\title{
Eltern von schwerkranken Kindern begleiten
}

\author{
Psychosoziale Unterstützung auf der pädiatrischen Intensivstation Wenn \\ ein Kind verunglückt oder eine lebenseinschränkende Diagnose erhält, steht \\ von einer Sekunde auf die andere die Welt für dessen Eltern still. Die Schlüssel- \\ frage "Überlebt mein Kind?" stellt das Klinikpersonal vor eine große Herausfor- \\ derung - denn nicht nur die mitunter hochmoderne intensivmedizinische Ver- \\ sorgung des Kindes, sondern auch die zwischenmenschliche psychosoziale \\ Betreuung der Eltern ist sehr anspruchsvoll. \& Carmen Eckerstorfer
}

\section{ZUSAMMENFASSUNG}

\begin{abstract}
Die Aufnahme eines Kindes auf die PICU (pädiatrische Intensive Care Unit) stellt für die Eltern eine traumatisierende Situation dar. Neben der Versorgung des kritisch kranken Kindes, begleitet und betreut das medizinische Fachpersonal auch die Eltern. Mithilfe einer vertrauensvollen Beziehung und in entlastenden und ressourcenaktivierenden Gesprächen wird den Eltern geholfen, aus der emotionalen Überlastung heraus wieder handlungsfähig zu werden. Durch authentisches Auftreten und mit einer klugen Portion Humor kann dieser Herausforderung meistens jedoch gut begegnet werden.
\end{abstract}

\section{Schlüsselwörter: pädiatrische Intensivstation, PICU, Elternbegleitung, psychosoziale Beratung, kritisch krankes Kind}

D ie Ursachen und Gründe, warum ein Kind auf einer pädiatrischen Intensivstation versorgt werden muss, sind vielfältig: Von angeborenen Fehlbildungen wie offener Bauchdecke oder Herzfehler über schwerwiegende Erkrankungen wie Tumore oder Infektionskrankheiten bis hin zu lebensbedrohlichen Unfällen wie Ertrinken oder Schädel-Hirn-Trauma durch Sturz oder Ähnliches. Die Art und Weise, wie Eltern von der Diagnose oder Erkrankung erfahren sind deshalb auch sehr verschieden von vorbereitet erwartend bis völlig überraschend kann alles zutreffen. Dementsprechend unterschiedlich sind auch die Reaktionen und Bedürfnisse der betroffenen Eltern in der ersten Zeit nach der Aufnahme auf der PICU (pediatric intensive care unit). Wie kann das Pflegepersonal Eltern in dieser Situation begleiten und ihnen nicht nur pflegerisch, sondern auch fachlich kompetent als Berater und Begleiter in dieser Krise gegenübertreten? Was brauchen Eltern in dieser Situation wirklich?

In einer qualitativen Studie haben Spitz-Köberich et al. (2010) die Bedürfnisse von Eltern mittels Interviews von betroffenen Müttern und Vätern untersucht. Gefühle wie Angst, Zweifel, Wut und völlige Hilflosigkeit sowie Reaktionen wie Schock, Erschöpfung und emotionaler Zusammenbruch sind nur ein sehr kleiner Einblick in die Welt der Betroffenen und ihrer Belastungen. Von zentraler Bedeutung war laut dieser Studie die Sorge um das kranke Kind. Weiter wurden sechs Bereiche herausgefiltert, die die Wünsche und Erwartungen der Eltern widerspiegeln: das Kind in guten Händen wissen, beim Kind sein dürfen, miteinbezogen werden, Fürsorge für sich und das Kind erfahren, informiert werden und Kontinuität erleben. Mit dem Wissen um diese Bedürfnisse und in Anlehnung an die "Grundprinzipien der Akutinterventionen"von Hausmann (2016) (Checkliste) sollen hier Empfehlungen für die psychosoziale Begleitung von betroffenen Eltern auf PICU zusammengetragen werden.

\section{Vertrauen schafft Ordnung im Gefühlschaos}

Die psychosoziale Unterstützung durch Pflegefachpersonen geschieht meist ganz "nebenbei“ - bei der Versorgung, der Verabreichung der Medikamente oder bei der Überprüfung der Vitalwerte. In diesen Situationen entstehen häufig ganz ungeplante aber sehr intensive Gespräche. Auch wenn das Pflegepersonal nicht immer für diese Gespräche geschult und darauf vorbereitet ist, ist es dennoch für betroffene Eltern und Familienangehörige oft sehr hilfreich, solche Entlastungsgespräche führen zu können, wie die retrospektive qualitative Inhaltsanalyse von Elternberichten bestätigt. Demnach stellt das Klinikpersonal mit 93\% die größte externe Ressource für Eltern dar (Fève \& Geipel 2020). Ausschlaggebend für den Aufbau einer vertrauensvollen Beziehung zwischen Eltern und 
dem Pflegepersonal ist Haltung, denn Haltung gibt Halt! Grundlegendes theoretisches Wissen über erwartbares Verhalten in Krisensituationen kann für viele Pflegefachpersonen sehr hilfreich und entlastend wirken, um mit der Bandbreite an Gefühlen und den oft rasch wechselnden Anforderungen umgehen und adäquat darauf reagieren zu können.

Das Konzept der personenzentrierten Beratung nach C. Rogers beruht auf der Grundannahme, dass jeder Mensch Experte für sich selbst ist und sämtliche Ressourcen zur Bewältigung seiner Lebenssituationen besitzt. Darauf aufbauend und mit Hilfe der Beratungsfähigkeiten der Akzeptanz, Empathie und Kongruenz können Pflegepersonen betroffene Eltern in dieser Ausnahmesituation begleiten und ihnen Halt geben.

Akzeptanz und unbedingte Wertschätzung ist das geduldige Annehmen, Akzeptieren und Respektieren der Reaktionen und Verhaltensweisen der betroffenen Eltern. Wichtig dabei ist das Bewusstsein über verschiedene Vorstellungen, Werte und Haltungen und erfordert eine starke Reflexionsfähigkeit. Es darf auch die eigene Betroffenheit gezeigt werden. Nichts irritiert mehr, als der distanzierte Umgang mit emotional belastenden Situationen. Mithilfe von einfühlsamem Verstehen ermöglichen Pflegende den Eltern ihren Schmerz zuzulassen und ihre Bedürfnisse wahrzunehmen. Um sich darauf einlassen zu können, ist nicht professionelle Dis$\operatorname{tanz}$, sondern professionelle Nähe besonders wichtig und entscheidend. Durch aktives Zuhören im Gespräch mit den Eltern wird nicht nur auf die verbale Aussage geachtet, sondern ganz bewusst die emotionale Botschaft erspürt. Dadurch fühlen sich Eltern ernstund wahrgenommen und können eine vertrauensvolle Beziehung zur Pflegeperson aufbauen. Durch gezieltes Nachfragen und durch das Anbieten von hilfreichen Handlungen werden im Gespräch oft gemeinsam Ressourcen gefunden und können so aktiviert werden. (Garten \& von der Hude 2019)

\section{Psychosoziale Begleitung von Eltern auf PICU}

Wird ein Kind akut auf der PICU aufgenommen, dreht sich in erster Linie alles um dessen medizinische Versorgung und Stabilisierung. Meist dürfen die Eltern während der Erstversorgung nicht mit im Raum sein. Wichtig ist es dann, sie regelmäßig über die aktuelle Situation und den Zustand ihres Kindes zu informieren. Beim Erstkontakt ist es wichtig, den betroffenen Eltern ruhig und sicher gegenüber zu treten. Man sollte sich mit Namen und Funktion vorstellen und in kurzen Sätzen erklären, was gerade geschieht.

Ist die Erstversorgung abgeschlossen und die Eltern dürfen zum Kind, kann der Anblick ihres kritisch kranken oder schwer verletzten Kindes sehr schmerzhaft und schwer auszuhalten sein. Daher sollten die Eltern bereits auf dem Weg zum Kind auf das veränderte Aussehen und die zahlreichen Kabel und Schläuche am Kind vorbereitet werden. Die wichtigsten Geräte können kurz erklärt werden, doch meist können Eltern diese Informationsflut neben ihren Emotionen gar nicht mehr verarbeiten. Wichtig ist jedoch, die Eltern über die zahlreichen Alarme und Warnsignale aufzuklären und so ausgelösten Stress zu reduzieren.

Grundsätzlich kann davon ausgegangen werden, dass Eltern in der ersten Zeit kaum Informationen aufnehmen können. Der Grund dafür sind Stresshormone, die in dieser Belastungssituation vermehrt produziert werden - das Gehirn befindet sich in der

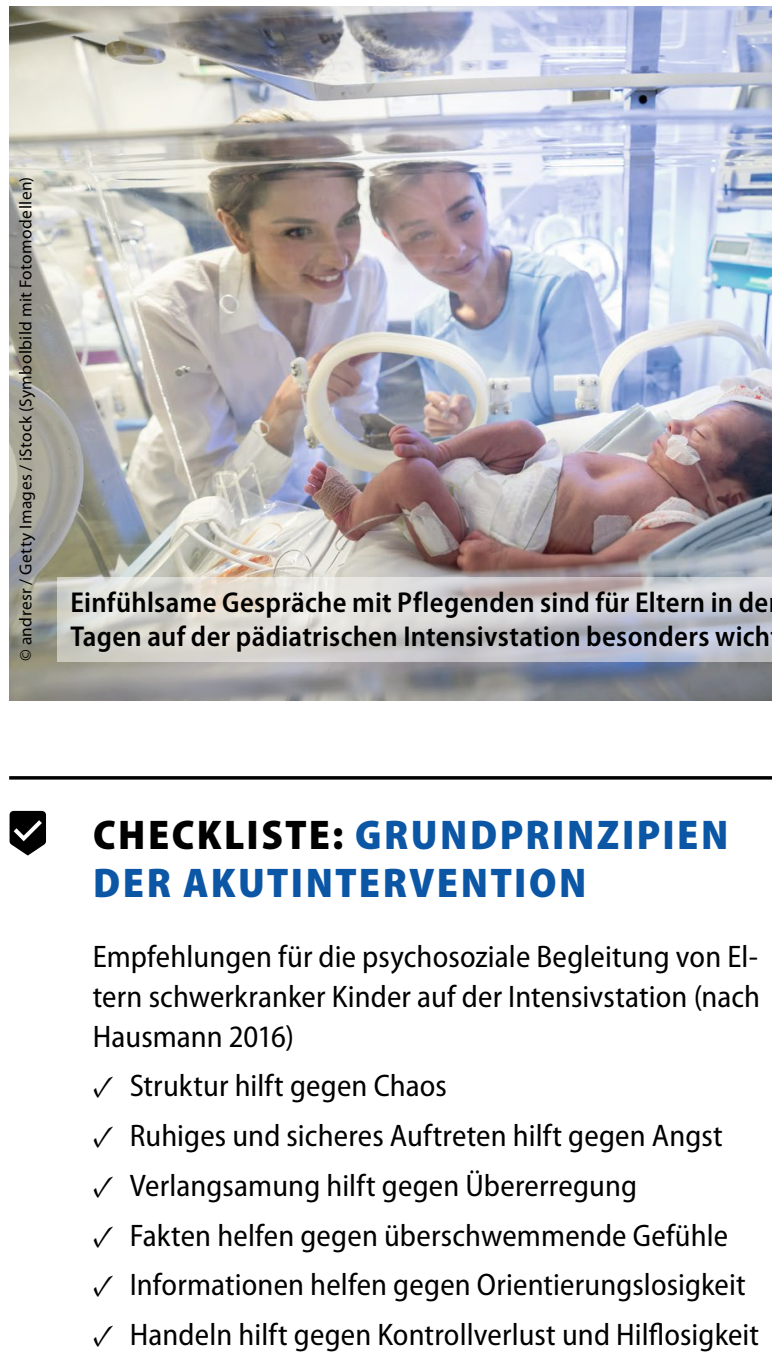

Zange, „normales“ Denken und Konzentration sind nur schwer möglich. Die Eltern funktionieren teilweise nur noch, fühlen sich „ver-rückt“. Dies ist ein Schutzmechanismus des Gehirns, da der komplette Schmerz ansonsten nicht auszuhalten wäre. Intensive Emotionen, aber auch das Fehlen von Emotionen ist normal. Wichtig in dieser Ausnahmesituation ist es, die Eltern vor zusätzlichen Reizen und Bedrohungen zu schützen, für einen sicheren Rahmen zu sorgen und für sie da zu sein. Durch wiederholende Informationen, Struktur und Verlangsamung kann die Übererregung reduziert werden und mit Hilfe von kleinen Aufgaben und Angeboten die Handlungsfähigkeit wiederhergestellt werden. Durch das Aktivieren des kognitiven Denkens kann die Belastung durch die überschwemmenden Gefühle ein wenig reduziert werden. Wichtig ist, die Verhaltensweisen, Reaktionen und Gefühle der betroffenen Eltern auszuhalten und vor allem sich weder persönlich noch fachlich angegriffen zu fühlen, denn häufig wird das medizinische Personal zur Projektionsfläche der elterlichen Emotionen.

Nichts fördert Angst und Unsicherheit mehr, als das Gefühl, dass Informationen verheimlicht oder Situationen nicht offen besprochen werden. Müssen schlechte Nachrichten überbracht oder 
kritische Entscheidungen getroffen werden, ist es ratsam, die Eltern nicht zu lange darauf warten zu lassen, denn meist spüren sie ohnehin, dass „etwas in der Luft liegt“ und das belastet sie zusätzlich. Damit Eltern die zahlreichen und stetig neuen Informationen verarbeiten können, muss ihnen ausreichend Zeit gegeben werden. Die Vermittlung in verständlicher Sprache und Wiederholungen können dies erleichtern. Wenn sie spüren, dass sie ernst genommen werden und all ihre Fragen, Ängste und Sorgen ansprechen können, kann dies enorm entlastend in dieser Ausnahmesituation sein.

\section{Nähe zum Kind ermöglichen}

Um Eltern die Anpassung an diese Ausnahmesituation zu erleichtern, hilft es, den Kontakt zum Kind zu fördern. Die Übernahme von kleinen Pflegehandlungen oder auch das Ermöglichen des Kuschelns, kann Eltern enorm helfen weitere Bewältigungsmechanismen zu aktivieren und Kraft und Energie für diese intensive Zeit zu tanken. Auch wenn es für manche undenkbar erscheint, ein intubiertes Kind mit unzähligen Zugängen und Drainagen, an lebenserhaltenden Maschinen und Medikamenten angeschlossen, den Eltern in den Arm zu geben, ist es doch möglich und kann Eltern und Kind Kraft geben. Diese Energie, diese Dankbarkeit, wenn Eltern nach Tagen oder sogar Wochen des Wartens, Hoffens und Bangens ihr Kind, vielleicht sogar das erste Mal, im Arm halten ist auch für Pflegepersonen unbezahlbar und lässt uns in schwierigen und belastenden Situation davon zehren.

\section{Die Trauerarbeit beginnt schon im Sterbeprozess}

Die Medizin heutzutage kann Wunder vollbringen und die Technik ermöglicht ganz unglaubliche Erfolge. Doch leider nicht immer. Trauerarbeit beginnt dann meist schon vor dem Tod - bei der Diagnosestellung oder beim Auftreten der ersten Krankheitssymptome und den damit verbundenen Ängsten. Lebensentwürfe werden zerstört, Pläne nicht erfüllt. Die beschränkte Lebenszeit und Konfrontation mit dem Tod setzt einen Prozess der Enttäuschung, Verzweiflung und des Loslassens in Gang. Neben der Hoffnung und dem Einsatz für das Überleben, muss aber auch Platz sein für das Tabuthema Sterben - mit all den dadurch ausgelösten Ängsten und Gefühlen.

Können Pflegepersonen den betroffenen Eltern eine vertrauensvolle Beziehung anbieten, einen sicheren Rahmen schaffen und offen für alle aufkommenden Gefühle sein, so können sich Eltern ihrer Hoffnung aber auch ihrer Angst vor dem Verlust des Kindes stellen und offen und ehrlich damit auseinandersetzen. Um sich auf diese Gefühle einlassen zu können, benötigen Eltern jedoch viel Ermutigung und Stärkung. Die Begleitung in dieser Pendelbewegung bedarf einer Atmosphäre von Sicherheit und Vertrauen, ohne Vorurteile und Wertung.

Verstirbt ein Kind auf der PICU, beginnt nach der vorangegangenen Hektik eine sehr ruhige Zeit für die Angehörigen. Je nach Wünschen und Bedürfnissen der Eltern, kann gemeinsam mit ihnen das Kind gewaschen und angekleidet, Erinnerungen wie Fußund Handabdrücke gemacht oder Erinnerungsbilder vom Sternenfotografen beauftragt werden. Das Begreifen des Todes findet in dieser Zeit durch den Kontakt zum verstorbenen Kind statt. Diese intensive Auseinandersetzung fördert den Trauerprozess und hilft, die schmerzhafte Realität anzunehmen. Außerdem ist es für ver- waiste Eltern hilfreich, nach einer gewissen Zeit noch einmal Kontakt mit dem betreuenden Personal aufzunehmen, um offene Fragen oder mögliche Missverständnisse zu klären oder auch um gemeinsam Erinnerungen zu teilen und dem verstorbenen Kind zu gedenken. Mittels einer „Gedenk-Karte“ an die Eltern kann das betreuende Team seine Anteilnahme ausdrücken und die Möglichkeit eines Anschlussgespräches anbieten.

Aber die PICU ist nicht nur ein trauriger Ort. Es ist wichtig, dass gerade hier auch Freude, Lachen und Humor zugelassen werden. Dies gilt es, angepasst an die Situation, zu ermöglichen und zu gestalten.

\section{FAZIT}

Wird ein Kind in einem kritischen Zustand auf der Intensivstation aufgenommen, gilt es neben der medizinischen und hochtechnischen Versorgung auch, eine auf Vertrauen basierende Beziehung zu den Eltern aufzubauen: Durch offenes und aktives Zuhören und Aushalten der elterlichen Gefühle.

Dabei gilt es für die Pflegenden, die psychischen Belastungen der Eltern einzuschätzen, individuelle Bedürfnisse zu erkennen und Ressourcen zu finden und zu fördern.

Eltern bevorzugen meist eine klare, ehrliche und vor allem eine verständliche Informationsweitergabe von Seiten des medizinischen Personals.

Die Literatur finden Sie online über das eMag der PflegeZeitschrift und auf springerpflege.de

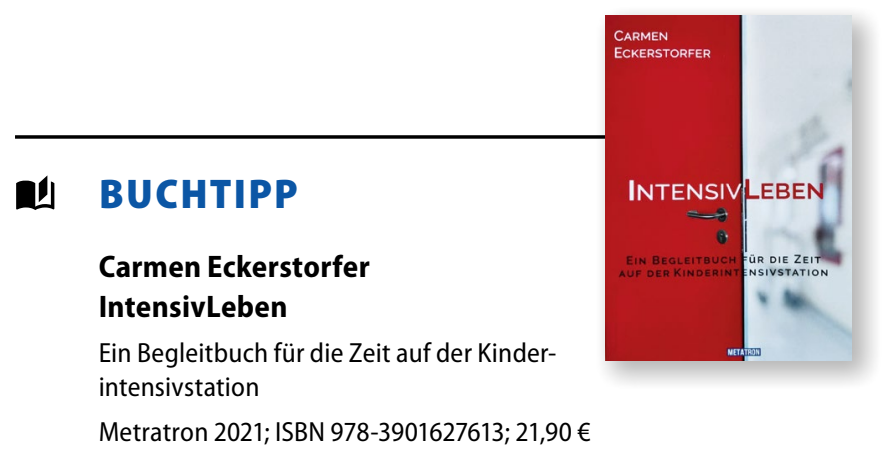

\section{Autorenkontakt}

Carmen Eckerstorfer, MSc, Kinderintensivkrankenschwester, psychosoziale Beraterin in freier Praxis, Schwerpunkt Begleitung von Familien bei Krankheit und Tod eines Kindes, und Vortrags- und Lehrtätigkeit.

E-Mail: info@carmen-eckerstorfer.at 
Hier steht eine Anzeige.

黛 Springer 